\title{
Ellis-Van-Creveld Syndrome and Congenital Cardiac Anomaly: Common Atrium with Atrioventricular Canal Septal Defect
}

\author{
Srikrishna Sirivella \\ Department of Cardiothoracic Surgery, Sri Venkateswara Institute of Medical Sciences, Tirupati, India \\ Email: sirivella@gmail.com
}

How to cite this paper: Sirivella, S. (2021) Ellis-Van-Creveld Syndrome and Congenital Cardiac Anomaly: Common Atrium with Atrioventricular Canal Septal Defect. World Journal of Cardiovascular Surgery, 11, 133-140.

https://doi.org/10.4236/wjcs.2021.1112017

Received: October 22, 2021

Accepted: December 26, 2021

Published: December 29, 2021

Copyright $\odot 2021$ by author(s) and Scientific Research Publishing Inc. This work is licensed under the Creative Commons Attribution International License (CC BY 4.0).

http://creativecommons.org/licenses/by/4.0/

\begin{abstract}
Background: Children presenting with physical features of chondro-ectodermal dysplasia (Ellis-Van Creveld syndrome) such as skeletal and joint abnormalities often have concomitant congenital cardiac anomalies. Presence of cardiorespiratory symptoms in children with Ellis-Van Craved syndrome warrants a thorough cardiologic evaluation to recognize and treat underlying congenital heart anomaly. Aim: A child with physical stigmata of Ellis-Van-Creveld syndrome is evaluated to detect an associated congenital cardiac anomaly and accomplish successful repair of the underlying cardiac lesion to reduce the cardiac related morbidity and improve the patient survival. Case Presentation: Ten years old boy with chondroectodermal dysplasia (dental anomalies, genu valgum and other skeletal abnormalities) presented with dyspnea and cyanosis. Cardiac evaluation by $2 \mathrm{D}$ echo revealed an atrioventricular (AV) canal septal defect with AV valve regurgitation and a common atrium. Angiocardiography showed a goose neck deformity of the left ventricular outflow tract. The Qp/Qs was 3.4: 1, with systemic arterial oxygen desaturation $\left(\mathrm{SaO}_{2}\right.$ of 0.7$)$ and $\mathrm{O}_{2}$ saturation in the common atrium was 0.7 . The pulmonary venous connections to the common atrium were anomalous. Atriotomy on cardiopulmonary bypass and on a cardioplegic arrest discerned a partial AV canal septal defect with a common bridging leaflet, clefts in septal leaflets of tricuspid and mitral vlalves, an incompletely closed interventricular communication, and a common atrium with highly anomalous pulmonary venous insertions well anterior $(8 \mathrm{~cm})$ to vena caval orifices. Intracardiac repair was performed with two patches of Goertex to partition the common atrium into the pulmonary and systemic venous chambers after repair of the partial AV canal septal defect. Patient required only a temporary afterload reduction with enalapril; otherwise patient had an uneventful postoperative course. At a 2-year follow-up, the child was well without AV valve regurgitation and had normal
\end{abstract}


biventricular function. Conclusion: A child with Ellis-Van-Creveld syndrome with skeletal abnormalities and dental anomalies had manifested with cardio-respiratory symptoms. Preoperative cardiac and intraoperative evaluation showed a common atrium with severely anomalous pulmonary venous connection and partial AV canal septal defect. Successful biventricular repair was accomplished by repairing the partial AV canal septal defect and partitioning the common atrium into left and right atrium by a complex atrial routing technique with two patches of Gore-Tex. On a follow-up at 2 years, the patient had adequate biventricular function without $\mathrm{AV}$ valve regurgitation.

\section{Keywords}

CHD (Congenital Heart Disease), Cyanotic CHD, Great Vessel Anomalies, CHD Miscellaneous, Atrioventricular Septal Defects, CHD and Valve Lesions

\section{Introduction}

Children with Ellis-Van-Creveld syndrome or chondroectodermal dysplasia often manifest growth retardation with association of skeletal abnormalities such as genu valgum, syndactaly, polydactaly, micrognathia, and dental anomalies. The association of a congenital cardiac lesion in Ellis-Van-Creveld syndrome (chondro-ectodermal dysplasia) suggests that cardiac defect may be due to a chromosomal disorder inherited by an autosomal dominant trait. The common atrium identified in a patient with (chondro-ectodermal dysplasia) also may suggest that this congenital cardiac anomaly may be due to a chromosomal disorder inherited by an autosomal dominant trait. Common (single) atrium may occur as an isolated entity or is associated with other congenital cardiac malformations such as ventricular septal defects, Tetralogy of Fallot, Ebstein's malformation of the tricuspid valve, heterotaxy syndrome, anomalous systemic and pulmonary venous connections, and atrioventricular (AV) canal defects [1] [2]. Patients with Ellis-Van Craved syndrome exhibiting cardiac and respiratory symptoms warrant thorough cardiologic evaluation to detect and treat an associated congenital cardiac anomaly.

We cite a summary of a child with Ellis-Van-Creveld syndrome (chondroectodermal dysplasia) presented with a common atrium, anomalous pulmonary venous connection, and partial AV canal septal defect, which was corrected by two patch atrial routing technique to accomplish a bi-ventricular repair.

\section{Case Presentation}

A 10-year-old boy with chondroectodermal dysplastic syndrome was admitted with recurrent respiratory tract infections, dysponea and mild cyanosis. An informed consent was obtained from the parents of the child for cardiac evaluation and necessary treatment. On physical examination the child exhibited stunted growth with skeletal abnormalities such as genu valgum, syndactaly, polydactaly, 
micrognathia, and dental anomalies. The heart was enlarged, the second heart sound was wide and fixed with an apical diastolic and a systolic murmur. Echocardiographic examination discerned a common atrium with atrioventricular canal defect, a common AV valve, and AVvalve septal leaflets with AV valve regurgitation (Figure 1). There was a left to right shunt at atrial level with presence of probable anomalous pulmonary venous connection. Cardiac catheterization data showed the Qp/Qs 3.4: 1 and the pulmonary vascular resistance of 2 wood units. The calculated oxygen saturation (Sao2) in the pulmonary veins was $99 \%$, but it was $70 \%$ in the common atrium, the pulmonary artery, and the aorta. Angiogocardiogram delineated AV canal defect with AV valve regurgitation, and gooseneck deformity of the left ventricular outflow tract (Figure 2).

Patient was placed on cardiopulmonary bypass using bicaval venous cannulation for venous drainage and ascending aortic cannulation for an arterial return. Intra-cardiac repair was performed under moderate systemic hypothermia with

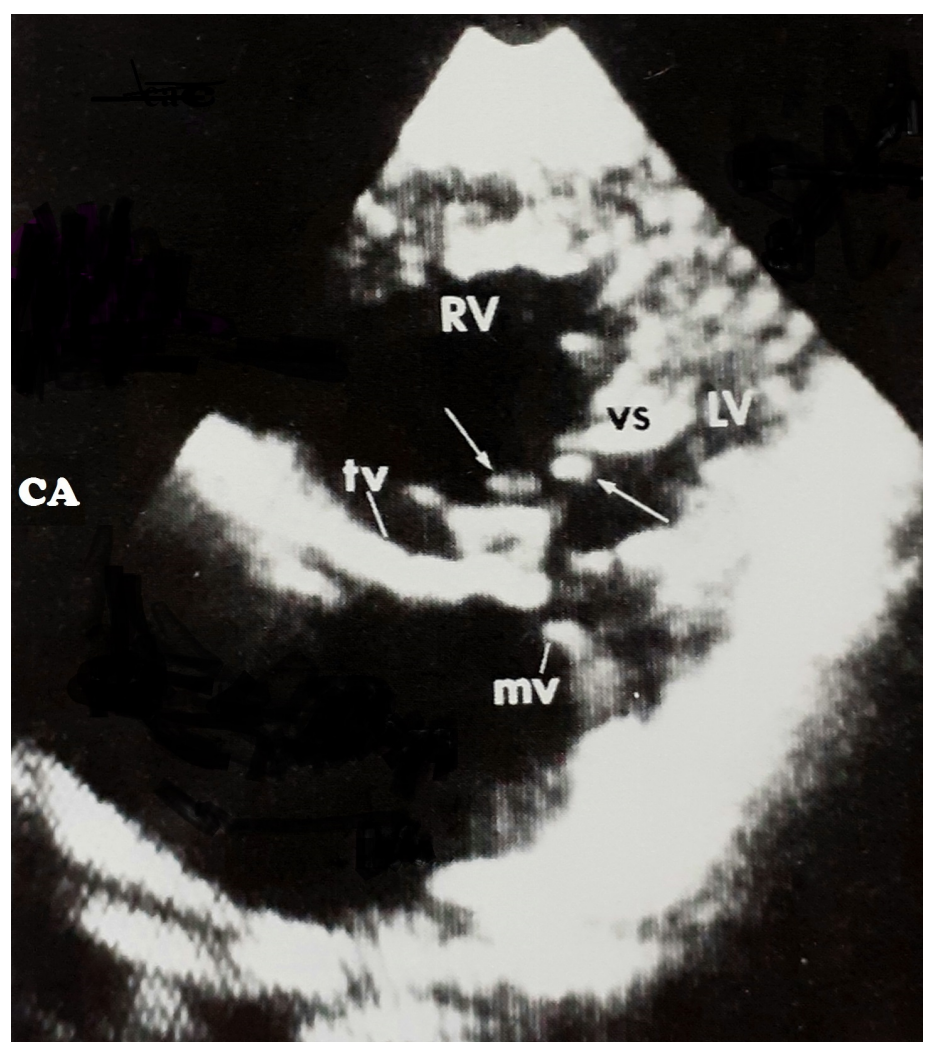

Figure 1. The cross sectional $2 \mathrm{D}$ echocardiogram in a child with Ellis-Van-Creved syndrome. The apical view demonstrates atrioventricular (AV) canal septal defect. The common anterior leaflet with tricuspid (right) and mitral (left) components appears to be attached to crest of the ventricular septum by multiple chordae tendinae (arrow heads). Note complete absence of the atrial septum above the AV valves suggesting presence of a common atrium. $\mathrm{CA}=$ common atrium, $\mathrm{LV}=\mathrm{left}$ ventricle, $\mathrm{mv}=$ mitral valve component of a common $\mathrm{AV}$ valve, $\mathrm{RV}=$ right ventricle, $\mathrm{tv}=$ tricuspid valve component of a common $\mathrm{AV}$ valve, VS $=$ ventricular septal crest. 


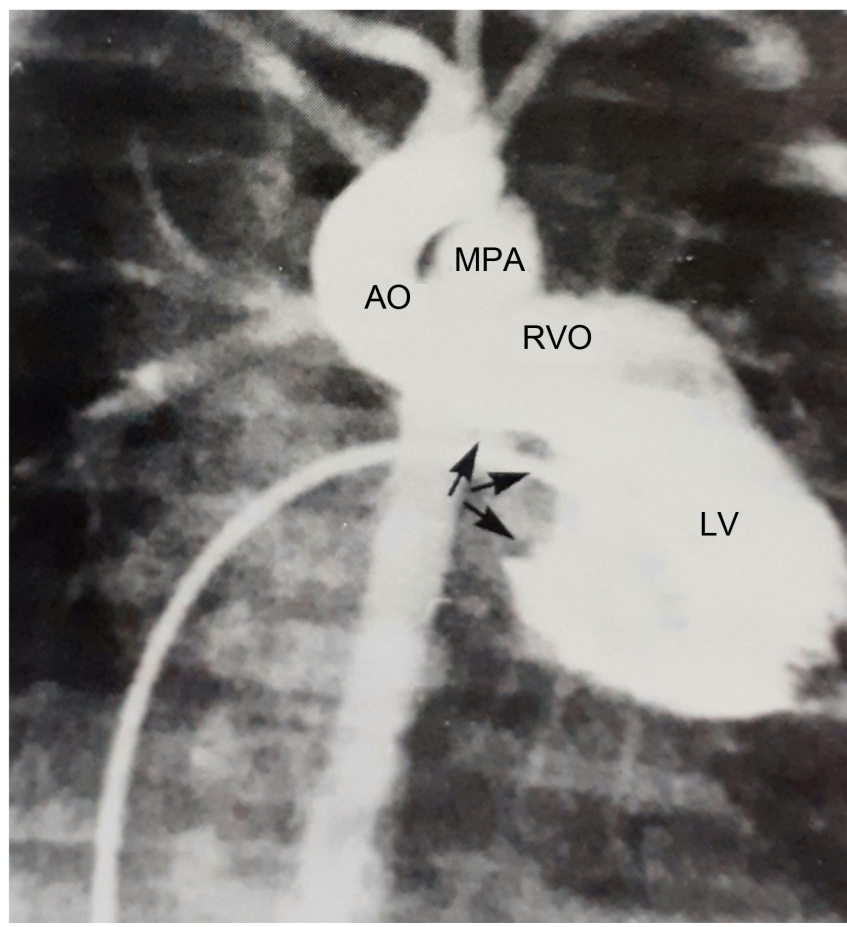

Figure 2. The left ventricular angiogram on posteroanterior view in a child with Ellis-Van-Creveld syndrome. It depicts a complete atrioventricular canal septal defect with the characteristic gooseneck deformity (arrow heads) of the left ventricular outflow tract due to downward displacement of the atrioventricular valve. Opacification of the right ventricular outflow tract and main pulmonary trunk prior to opacification of atria suggests presence of an interventricular communication. $\mathrm{AO}=$ ascending aorta, $\mathrm{LV}=\mathrm{left}$ ventricular outflow tract. $\mathrm{MPA}=$ main pulmonary artery, $\mathrm{RVO}=$ right ventricular outflow tract.

a cold blood cardioplegic arrest. Through a vertical atriotomy incision the morphology of the congenital anomaly was examined. The examination has revealed a common atrium without any remnants of atrial septal tissue. There were clefts in the septal leaflets of both the left and the right atrioventricular (AV) valves with a thin bridge of normal valve tissue separating both the AV valves. Beneath the septal leaflets of the AV valves, a small $(0.8$ to $0.9 \mathrm{~cm})$ defect was present in the ventricular septum and was partially covered by a membrane which was thin, fibrous, and with an aneurysmal bulge. The defect in the ventricular septum was closed with interrupted pledgeted sutures of 5/0 polypropylene incorporating the overhanging fibrous tissue within the sutures. Cleft in leaflets of both atrioventricular valves were repaired with interrupted $6 / 0$ polypropylene sutures. The connection of the pulmonary veins into the atrium were found to be severely anomalous, i.e., they were inserted into the atrium in much anterior $(8 \mathrm{~cm})$ in reference to the openings of both vena cava which were more posteriorly displaced and were in a plane closer to the AV valve. The coronary sinus ostial opening into the atrium was also more posteriorly displaced than usual. 
The common atrium was partitioned using two patches of Gore-Tex to separate it into approximately equal portions of systemic and pulmonary venous chambers committed to the tricuspid and mitral valve respectively (Figure 3). The first patch of Gore-Tex was first sutured to the bridge between two atrioventricular valves, with a double armed 5/0 polypropylene suture, the suture beginning in the mid portion of the AV valve bridge. After separating the AV valves, the suture line was carried out on either direction suturing the patch to the wall of the body of the atrium until the orifices of the superior and inferior vena cava were reached. The suturing of the patch was continued on the inferior margins of the orifices of the superior and inferior vena cavae, then past the vena caval orifices to the atrial wall. Here, the sutures were tagged. A second patch of Gore-Tex was anchored to free margin of this first patch at this level with a separate suture of a double armed 5/0 polypropylene, the suture beginning at the middle and was continued both superiorly and inferiorly and were tied to the tagged sutures. The superior margin of the second patch was tailored and sutured to the wall of atrium from the orifice of the superior veana cava curving it initially upward and posteriorly towards the roof of the atrial wall and then curving it downward and anteriorly to reach the ostium of the right superior pulmonary vein. Similarly, the inferior margin of the second patch was tailored and sutured to the wall of the atrium from the orifice of the inferior vena cava curving it downward and posteriorly, then curving it upward and anteriorly to reach the ostium of the right inferior pulmonary vein. At the ostia of the pulmonary veins both the sutures were tagged. Both the tagged sutures were then continued to suture the anterior margin of the patch to the anterior wall of ostia of both the right superior and inferior pulmonary veins and the anterior atrial wall until midpoint of the patch was reached. Here both the sutures were tied (Figure 3). This procedure performed such partitioned the common atrium into approximately equal portions of the systemic and the pulmonary venous chambers. The coronary sinus ostium was recruited into the pulmonary venous atrium to prevent damage to the atrioventricular node. The atriotomy incision was closed in the usual fashion. The patient was successfully weaned of cardiopulmonary bypass with a stable hemodynamics.

The care of the patient postoperatively was followed by the established standards of cardiorespiratory care. Patient was maintained on mechanical ventilation for the first 24 hours. In addition to heart rate and cardiac rhythm, monitored were the mean systemic arterial pressure, central venous pressure, and the left atrial pressure and these were maintained within normal range. In addition, the chest tube drainage, volume status of the patient and urine output and peripheral tissue perfusion were closely monitored. The tissue perfusion is maintained adequately with infusion of adequate volume, and administration of an infusion of doubutamine at $5 \mathrm{mcg} / \mathrm{kg} / \mathrm{minute}$ for 72 hours. Post-repair systemic arterial oxygen saturation was $97 \%$. There was no evidence of a leak from the baffle without any evidence of AV valve regurgitation on postoperative echocardiographic examination. Patient required a 10 day period of afterload reduction 


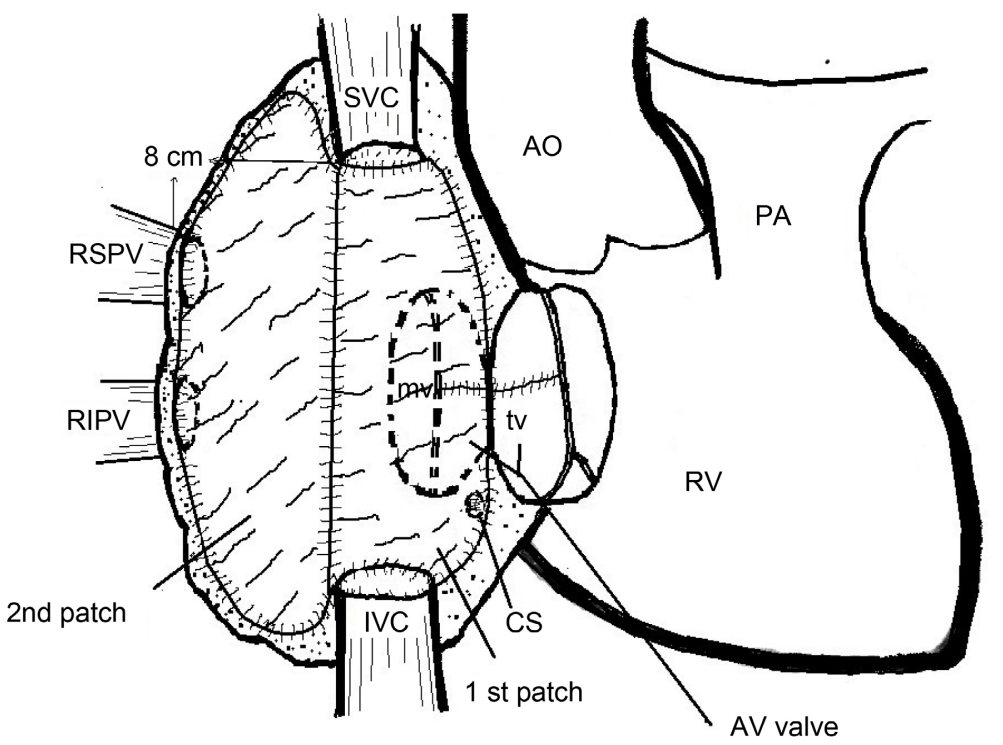

Figure 3. The diagram showing repair of a partial AV (atrioventricular) canal septal defect and partition of a common atrium into equal sizes of systemic and pulmonary venous atrial chambers using two patches of Goertex. Note also severely anomalous right pulmonary venous connections into the common atrium $8 \mathrm{~cm}$ anterior to the both vena caval orifices. Initially, the clefts in the leaflets of mitral and tricuspid components were repaired, as well as a small ventricular septal defect. The common AV valve was separated into the tricuspid and the mitral valve components by anchoring a $1^{\text {st }}$ patch of Goertex to the bridge of the leaflet tissue of the common AV valve. The suturing of the rest of the margin of the patch was continued both superiorly and inferiorly to anchor the posterior atrial wall and posterior margins of the ostia of the SVC and IVC respectively. The patch was tailored as the suturing was proceeded. The second patch of Goertex was anchored to the 1st patch of Goertex at the level of ostia of both vena cava. From SVC orifice, the second patch was tailored and the margin of the patch was sutured to the posterior atrial wall, the suture line running upward and posteriorly towards the roof of the atrium, then the suture line was curved gently downward and anteriorly anchoring the patch to the anterior atrial wall up to the orifice of the right superior pulmonary vein. From the IVC orifice the second patch was tailored and the margin of the patch was sutured to the posterior atrial wall, the suture line running downward and posteriorly towards the floor of the atrium, then the suture line was gently curved upward and anteriorly anchoring the patch to the anterior atrial wall up to the orifice of the right inferior pulmonary vein. Last, the rest of the margin of the $2^{\text {nd }}$ patch was approximated to the anterior margins of the both pulmonary venous ostia and intervening anterior atrial wall between pulmonary venous ostia. This procedure, thus recruits both pulmonary veins into the left atrium and vena cava into the systemic venous atrium, partitioning the common atrium into equal chambers in size. $\mathrm{AO}=$ ascending aorta, $\mathrm{AV}$ valve $=$ atrioventricular valve, $\mathrm{CS}=$ coronary sinus, $\mathrm{IVC}=$ inferior vena cava, $\mathrm{mv}=$ mitral valve component, $\mathrm{PA}=$ pulmonary artery, $\mathrm{RSPV}=$ right superior pulmonary vein, $\mathrm{RIPV}=$ right inferior pulmonary vein, $\mathrm{RV}=$ right ventricle, $\mathrm{SVC}=$ superior vena cava, $\mathrm{tv}=$ tricuspid valve component.

with ACE inhibitor, enalapril (5 mg/day) to maintain adequate peripheral perfusion and warm extremities after termination of infusion of doubutamine. The rest of the postoperative course was uneventful and the patient was discharged with a low dose enalapril $(2.5 \mathrm{mg} /$ day $)$ for a period of another 4 weeks. The patient was followed monthly for a few months, then every 6 months. At 2-year 
follow-up the patient was well without any evidence of $\mathrm{AV}$ valve regurgitation or a leak from the atrial baffle and had demonstrated good biventricular function.

\section{Comment}

In common atrium, the heart is a tripartite chamber (tricameral heart) consisting of morphologic right and left ventricles and a single atrium due to arrest in the development of atrial septum. In necropsy studies of the congenital hearts, the common atrium represented only $0.3 \%$ to $0.5 \%$ of the cases, and in approximately $2 \%$ of the specimens of atrial septal defects there was a complete absence of the atrial septum. [3]. It is usually associated with a cleft in mitral valve but without an interventricular communication and is considered to be a variant of atrioventricular canal deformity or it may be associated with normal atrio-ventricular valves. [2] [3]. In a recent classification, the Society of Congenital Heart Surgeons included a single atrium under developmental anomalies of the endocardial cushions or atrio-ventricular valves. The morphological findings in this patient support this classification as there was an associated small ventricular septal defect and clefts in the both AV valves due to incomplete fusion of the endocardial cushions. The association of common atrium in Ellis-Van Creveld syndrome (chondro-ectodermal dysplasia) suggests that the cardiac defect may be a chromosomal disorder inherited by an autosomal dominant trait [4]. Patient presenting with respiratory symptoms in Ellis-Van Craved syndrome warrants thorough cardiologic evaluation to detect and treat an associated cardiac anomaly.

The morphology of common atrium is either of a right or a left atrium, or both atrial morphologies may also coexist. The pulmonary and systemic venous connections to the common atrium are either normal or anomalous [5]. The hemodynamic burden imposed by common atrium is similar to that of a large atrial septal defect and there is free mixing of systemic and pulmonary venous blood [2] [3]. The resistance of pulmonary vascular bed varies and is either normal or is elevated to moderate levels.

Surgical correction of common atrium associated with partial atrioventricular canal involves repair of atrioventricular valves and complete atrial septation [6]. Atrial septation may be technically challenging if the pulmonary veins are severely anomalous as observed in this case. In complex atrial routing technique which requires undue curvature on the patch for separation of systemic and pulmonary venous connections, use of a rigid material such as Gore-Tex and two patch technique may prevent from distortion and obstruction of the venous orifices, and adequate partitioning of both the systemic and pulmonary venous chambers is important to prevent undue alterations in atrial compliance. Failing this technique, due to severely anomalous pulmonary venous connections, revision of the patch may be required or a modified Fontan correction may be an alternate surgical option if atrioventricular valve competence can be restored. The results of atrial septation procedure for surgical correction of common atrium vary with an early mortality ranging from 0 to $20 \%$ [3]. This case represents a 
successful attempt at complex atrial routing technique to achieve a bi-ventricular repair.

\section{Conclusion}

In conclusion, a child with Ellis-Van-Creveld syndrome with physical stigmata of retarded growth and skeletal abnormalities such as genu valgum, syndactaly, polydactaly, micrognathia, and dental anomalies had manifested with cardio-respiratory symptoms. Common atrium with severely anomalous pulmonary venous connection and partial AV canal septal defect was recognized as an associated congenital cardiac anomaly. Successful biventricular repair was accomplished by repairing the partial AV canal septal defect and partitioning the common atrium into an equal size of left and right atrium by a complex atrial routing technique with two patches of Gore-Tex. On follow-up at 2 years, the patient had an adequate biventricular function.

\section{Conflicts of Interest}

The author declares no conflicts of interest regarding the publication of this paper.

\section{References}

[1] Digilio, M.C., Marino, B., Ammirati, A., Borzaga, U., Giannotti, A. and Dallapiccola, B. (1999) Cardiac Malformations in Patients with Oral-Facial-Skeletal Syndromes: Clinical Similarities with Heterotaxia. American Journal of Medical Genetics, 84, 350-356.

https://doi.org/10.1002/(SICI)1096-8628(19990604)84:4\%3C350::AID-AJMG8\%3E3 .0.CO;2-E

[2] Munoz-Armas, S., Gorrin, J.R., Anselmi, G., Hernandez, P.B. and Anselmi, A. (1968) Single Atrium. Embryologic, Anatomic, Electrocardiographic and Other Diagnostic Features. American Journal of Cardiology, 21, 639-652. https://doi.org/10.1016/0002-9149(68)90261-0

[3] Rastelli, G.C., Rahimtoola, S.H., Ongley, P.A. and McGoon, D.C. (1968) Common Atrium: Anatomy, Hemodynamics, and Surgery. Journal of Thoracic and Cardiovascular Surgery, 55, 834-841. https://doi.org/10.1016/S0022-5223(19)42898-5

[4] Blackburn, M.G. and Belliveau, R.E. (1971) Ellis-Van Creveld Syndrome-A Report of Previously Undescribed Anomalies in Two Siblings. American Journal of Diseases of Children, 122, 267-270. https://doi.org/10.1001/archpedi.1971.02110030125024

[5] Ghosh, P.K., Donnelly, R.J., Hamilton, D.I. and Wilkinson, J.L. (1977) Surgical Correction of a Case of Common Atrium with Anomalous Systemic and Pulmonary Venous Drainage. Journal of Thoracic and Cardiovascular Surgery, 74, 604-606. https://doi.org/10.1016/S0022-5223(19)40888-X

[6] Danielson, G.K., McMullan, M.H., Kinsley, R.H. and DuShane, J.W. (1973) Successful Repair of Complete Atrioventricular Canal Associated with Dextroversion, Common Atrium and Total Anomalous Systemic Venous Return. Journal of Thoracic and Cardiovascular Surgery, 66, 817-822.

https://doi.org/10.1016/S0022-5223(19)40576-X 